\title{
DEXTERITY OPTIMIZATION OF KINEMATICALLY REDUNDANT MANIPULATORS IN THE PRESENCE OF JOINT FAILURES
}

\author{
Christopher L. Lewis and Anthony A. Maciejewski $\dagger$ \\ School of Electrical Engineering, Purdue University, West Lafayette, IN 47907, U.S.A.
}

(Accepted in final revised form 30 March 1993)

\begin{abstract}
Robotic manipulators working in remote or hazardous environments require additional measures to ensure their usability upon the failure of an actuator. This work considers failure modes that result in an immobilized joint and uses the concept of worst-case dexterity to define kinematic and dynamic fault tolerance measures for redundant manipulators. These measures are then used to specify the operating configuration which is optimal in the sense that the manipulator's dexterity remains high even if one of its joints fails in a locked position. The close relationship between fault tolerance and dexterity is examined using a simple planar manipulator as an example. It is demonstrated that an inverse kinematic function which maintains a high level of fault tolerance also keeps the manipulator in well-conditioned configurations known to have desirable properties.
\end{abstract}

Key Words: Kinematically redundant, fault tolerance, dexterity, robotics.

\section{INTRODUCTION}

The use of robotic manipulators in remote and/or hazardous environments greatly increases the cost and in some cases eliminates the possibility of repairing failed actuators [1-3]. For this reason kinematically redundant manipulators have been proposed for these applications. The additional degrees of freedom permit a degree of dexterous motion of the end effector even after an actuator has failed and been immobilized. The extra degrees of freedom may also be used, prior to failure, to maintain configurations that minimize the loss of dexterity resulting from the failure of an actuator $[4,5]$. To optimize this type of fault tolerance for redundant manipulators, the notion of dexterity must be quantified.

Heuristically, the dexterity of a manipulator is described as the ease in which motion of the end effector can be accomplished. Since end effector velocity is controlled indirectly through the joint rates, the notion of kinematic dexterity is defined as a quantification of the relationship between the joint rates and the end effector's velocity. Taking this relationship one step further, the notion of dynamic dexterity is a quantification of the relationship between the actuator torques and the end effector's acceleration. This paper will first investigate the various measures of kinematic and dynamic dexterity. Then, to form measures of fault tolerance, these dexterity measures will be adapted to include the additional assumption that any one of the manipulator's actuators could fail and become locked. The minimum reduced kinematic or dynamic dexterity resulting from a single joint failure is defined to be the measure of kinematic or dynamic fault tolerance. A manipulator configuration which retains a high dexterity for all possible single joint failures is said to be fault tolerant. Using these measures of fault tolerance a three-link planar manipulator is examined and comparisons are drawn between these two measures.

The remainder of this paper is organized in the following manner. In Section II the kinematic and dynamic equations of motion for robotic manipulators are used to define kinematic and dynamic dexterity measures. Then, in Section III, these concepts of dexterity are extended to consider an arbitrary single joint failure and to form measures of kinematic and dynamic fault tolerance. With these measures in hand, Section IV examines the fault tolerance of a 3 degree-of-freedom (DOF) planar manipulator. It is shown that the kinematic singularities of the 
resulting manipulator Jacobians following a joint failure are the single most defining feature of both the kinematic and dynamic fault tolerance measures. Next, in Section V, optimal kinematic and dynamic fault tolerance inverses are proposed which select the optimally fault tolerant configuration from the set of joint configurations that achieve the desired end-effector position. In Section VI the kinematic fault tolerance inverse is compared to the pseudoinverse along a test trajectory to evaluate the performance both before and after a failure. Finally, conclusions are presented in Section VII.

\section{KINEMATIC AND DYNAMIC DEXTERITY}

The mathematical relationship between the joint rates, $\dot{\boldsymbol{\theta}}$, and the end-effector velocity, $\dot{\mathbf{x}}$, is given by the linear Jacobian equation

$$
J \dot{\theta}=\dot{\mathbf{x}}
$$

The Jacobian, $J \in R^{m \times n}$, like all matrices, may be decomposed using the singular value decomposition (SVD) so that

$$
J=U \Sigma V^{T}
$$

where $U \in R^{m \times m}$ and $V \in R^{n \times n}$ are orthogonal matrices and $\Sigma \in R^{m \times n}$ is a diagonal matrix consisting of the singular values, denoted $\sigma_{i}$, which are typically given in descending order. The SVD representation of $J$ is convenient due to the physical interpretation of the singular values and vectors. The singular values define the size of the possible motions of the end effector due to any given joint rate having a unit norm. The right singular vectors, $\hat{\mathbf{v}}_{i}$, describe the direction, or normalized linear combination of joint rates, which cause a motion of norm $\sigma_{i}$ in the $\hat{\mathbf{u}}_{i}$ direction of the workspace.

The singular values have been used to form a variety of kinematic dexterity measures for manipulators. For instance, the ratio of the largest: smallest singular value is the condition number of $J[6,7]$. Physically, it is clear that the condition number is a measure of the eccentricity of the velocity ellipsoid. Another popular measure is the product of all of the singular values, the manipulability, which is proportional to the volume of the velocity ellipsoid [8]. The sum of the singular values of $J$ has also been used as a dexterity measure. If the trajectory were known a priori then the distance to the edge of the ellipsoid in the direction of the known trajectory is a path specific dexterity measure. Finally, the smallest singular value of the Jacobian, $\sigma_{m}$, is a measure of the worst-case scaling of the mapping from joint velocities to end-effector velocities. Throughout the remainder of this work the worst-case kinematic dexterity, denoted by $\mathrm{km}$ and defined as

$$
k m=\sigma_{m}(J),
$$

will be used as a measure of kinematic dexterity. Unfortunately the relationship between actuator torques and end effector acceleration is not as simple. Following the development in [9], the dynamic equations of motion for robotic manipulators are generally given by

$$
M(\boldsymbol{\theta}) \ddot{\boldsymbol{\theta}}+\mathbf{h}(\boldsymbol{\theta}, \dot{\boldsymbol{\theta}})+\mathbf{g}(\boldsymbol{\theta})=\boldsymbol{\tau}
$$

where $M(\theta) \in R^{n \times n}$ is the moment of inertia matrix, $\mathbf{h}(\boldsymbol{\theta}, \boldsymbol{\theta}) \in R^{n}$ is a vector of torques caused by the centrifugal and Coriolis forces, $g(\theta) \in R^{n}$ is the vector of torques due to gravity and $\tau$ is the resulting vector of joint torques. It is well known that the inertia matrix, $M(\theta)$, for realistic systems is at all times invertible. The relationship between joint accelerations and the end-effector acceleration is given by

$$
J \ddot{\theta}+\dot{J} \ddot{\theta}=\ddot{x}
$$

which is obtained by differentiating (1). Now since we are concerned with analyzing the failure tolerant properties of manipulator configurations, the manipulator can be considered to be at rest so that $\dot{\theta}$ will be zero. Therefore, by combining (4) and (5), one obtains 
which describes the mapping between joint torques and end-effector acceleration. Noting that $J M^{-1} \mathrm{~g}$ is a uniquely determined vector at every point in the joint space, the applied torque to end-effector acceleration mapping may be characterized in a manner that is analogous to the kinematic mapping. Note, however, that the acceleration ellipsoid is shifted so that it is centered at $J M^{-1} \mathbf{g}$. If the manipulator is in motion, the Coriolis and centrifugal forces have a similar shifting effect on the acceleration ellipsoid, however, neither gravity nor Coriolis forces change the shape of the ellipsoid. Therefore, the worst-case dynamic dexterity, $d m$, is defined as the length of the smallest axis of the acceleration ellipsoid and is given by

$$
d m=\sigma_{m}\left(J M^{-1}\right)
$$

\section{A DEFINITION OF FAULT TOLERANCE}

The kinematic and dynamic dexterity measures defined in the previous section will now be extended to form a measure of fault tolerance for redundant manipulators. It will be assumed that failed actuators can be locked in their failed position. Fault tolerance may thus be quantified in terms of the velocity and acceleration ellipsoids for the resulting manipulator after the failed joint is locked. The minimum remaining dexterity over all possible joint failures is defined to be the measure of fault tolerance.

In the kinematic case, the Jacobian that relates the joint rates of the manipulator to the end-effector velocity with the $f$ th actuator locked, denoted by ${ }^{f} J$, is given by

$$
{ }^{f} \boldsymbol{J}=\left[\boldsymbol{j}_{1} \ldots \boldsymbol{j}_{f-1} \boldsymbol{j}_{f+1} \ldots \boldsymbol{j}_{n}\right]
$$

where the column $\boldsymbol{j}_{f}$ has been removed and where the remaining $\boldsymbol{j}_{i}$ s are identical to their values in $J$. The kinematic fault tolerance measure, denoted $\mathrm{kfm}$, can now be given in terms of the failure Jacobians as

$$
k f m=\min _{f=1 \ldots n} \sigma_{m}\left({ }^{f} J\right)
$$

The dynamic fault tolerance measure is quite similar in structure to the kinematic measure. It is the minimum of the dynamic measures of the system reduced by locking one of the joints. The dynamic equations of motion for a manipulator with the $f$ th actuator locked have the form

$$
{ }^{f} \boldsymbol{M}^{f} \ddot{\boldsymbol{\theta}}+{ }^{f} \boldsymbol{h}+{ }^{f_{\boldsymbol{g}}}={ }^{f_{\boldsymbol{\tau}}}
$$

where ${ }^{f} M$ is the inertia tensor of the original system with the $f$ th row and column removed, ${ }^{f} \boldsymbol{\theta}$ is the vector of the remaining joint variables, and ${ }^{f} \boldsymbol{h}$ is the Coriolis and centrifugal forces arising from the dependence of $M$ on $\boldsymbol{\theta}$ and is algebraically similar to $\boldsymbol{h}$ with $\dot{\theta}_{f}$ set to zero and the $f$ th element eliminated. The gravitational force, ${ }_{g} \boldsymbol{g}$, is $g$ with the $f$ th element eliminated and ${ }^{f} \tau$ is the vector of remaining joint torques. A dynamic fault tolerance measure, denoted $d f m$, may be defined from the dynamic dexterity of the reduced system as

$$
d f m=\min _{f=1 \ldots n} \sigma_{m}\left({ }^{f} J^{f} M^{-1}\right)
$$

\section{A SIMPLE PLANAR EXAMPLE}

In this section, the fault tolerance of a three-link planar manipulator is studied. All three links are chosen to have lengths of $1 \mathrm{~m}$ and are modeled as thin rods. Two different mass ratios are compared. In the first case, all link masses are $1 / 3 \mathrm{~kg}$. In the second case, the first link has a mass of $0.8 \mathrm{~kg}$ and second and third links have masses of $0.1 \mathrm{~kg}$. Note that the mass ratios are varied since $d m$ is inversely related to the total mass. The kinematic and dynamic dexterity and fault tolerance measures were evaluated throughout the joint space. The results are given in Figs 1-3 as contour plots in configuration space (c-space). Note that only the $\theta_{2}-\theta_{3}$ plane needs to be considered since all of the measures are independent of $\theta_{1}$. 

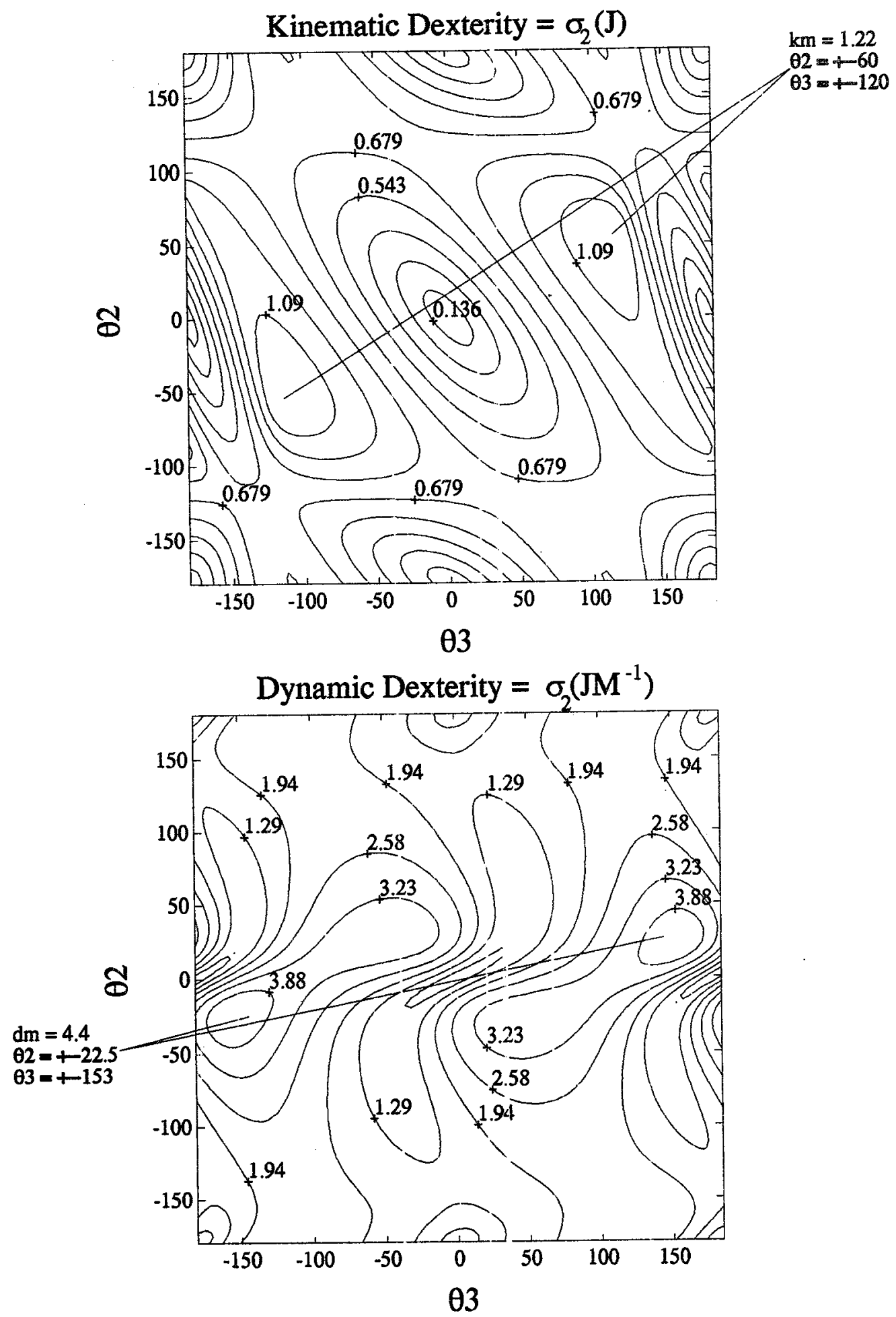

Fig. 1. The worst-case kinematic and dynamic dexterity measures for a 3 DOF planar manipulator are plotted as a function of the manipulator's configuration. The worst-case kinematic and dynamic dexterity are defined as the minimum singular value of $J$ and $J M^{-1}$, respectively. Only the $\theta_{2}-\theta_{3}$ plane of c-space is shown since these dexterity measures are independent of $\theta_{1}$. The manipulator's links are all identical with lengths of $1 \mathrm{~m}$, masses of $1 / 3 \mathrm{~kg}$, and are modeled as thin rods. 

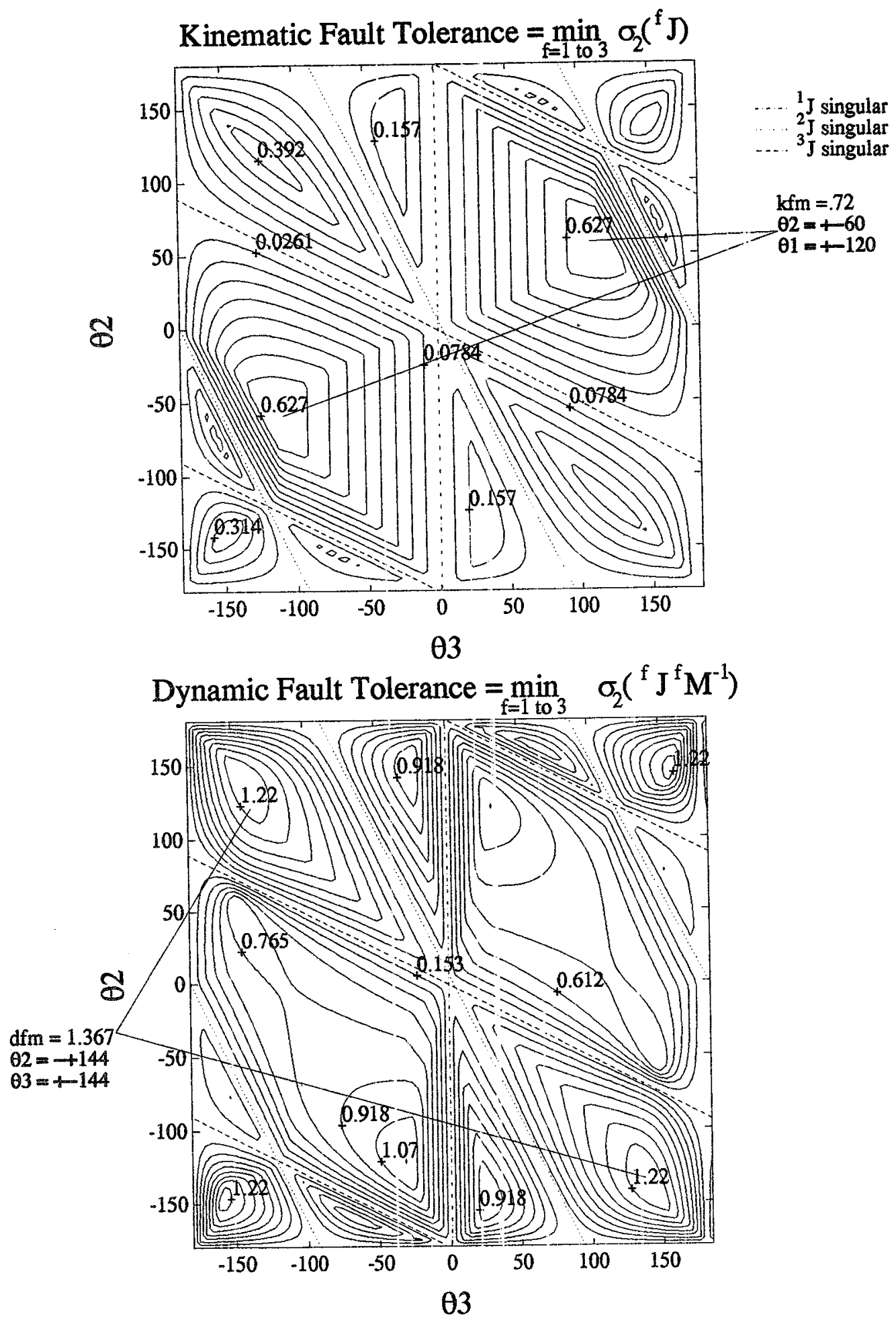

Fig. 2. The kinematic and dynamic fault tolerance measures for a 3 DOF planar manipulator are plotted as a function of the manipulator's configuration. The worst-case kinematic and dynamic fault tolerance are defined as the minimum remaining kinematic and dynamic dexterity, respectively, over all possible joint failures. Only the $\theta_{2}-\theta_{3}$ plane of c-space is shown since these measures are independent of $\theta_{1}$. The manipulator's links are all identical with lengths of $1 \mathrm{~m}$, masses of $1 / 3 \mathrm{~kg}$, and are modeled as thin rods. Regions of high fault tolerance are bounded by singularities in one or more of the Jacobians that would result from a joint failure. Note that the manipulator kinematics defines the boundaries of the dynamically fault tolerant regions. 


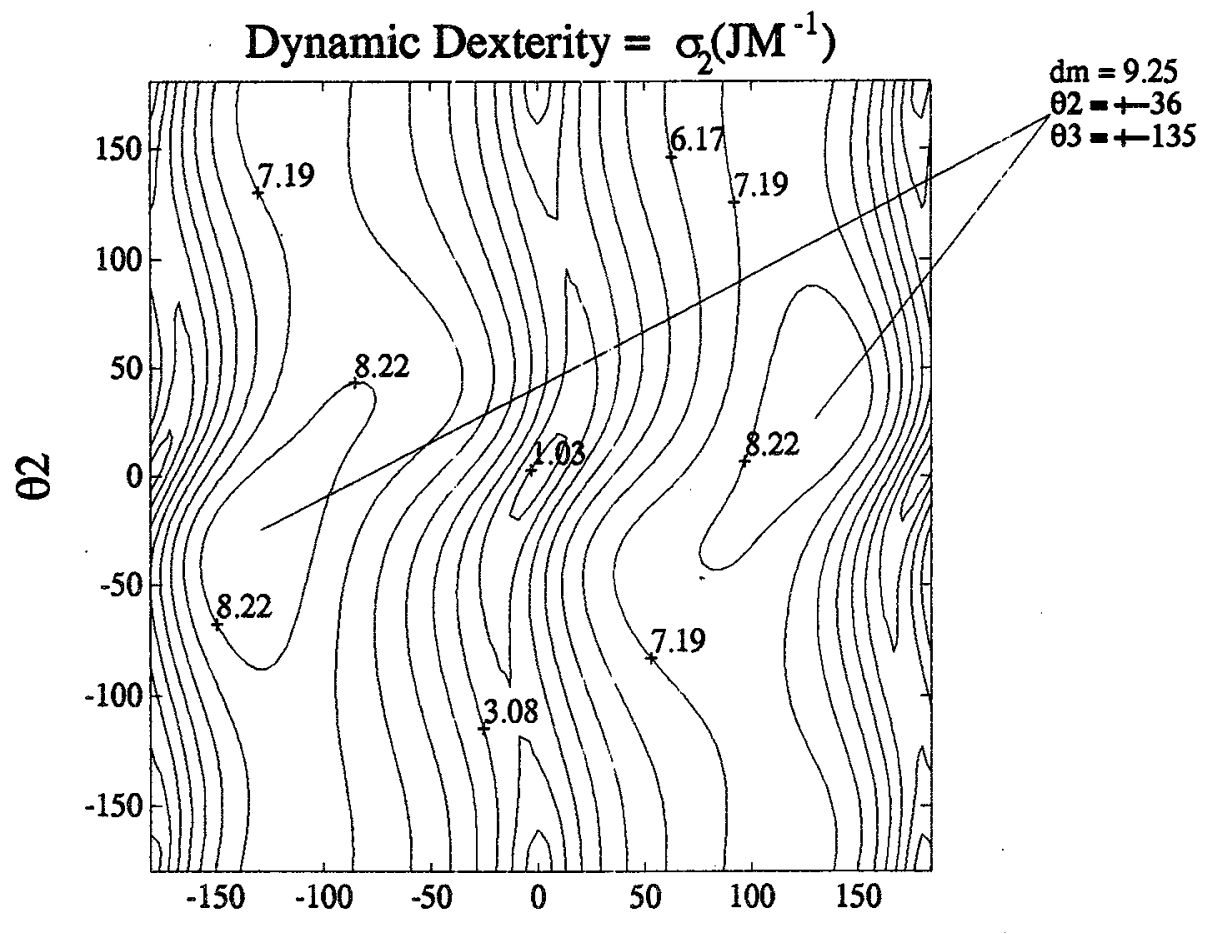

$\theta 3$

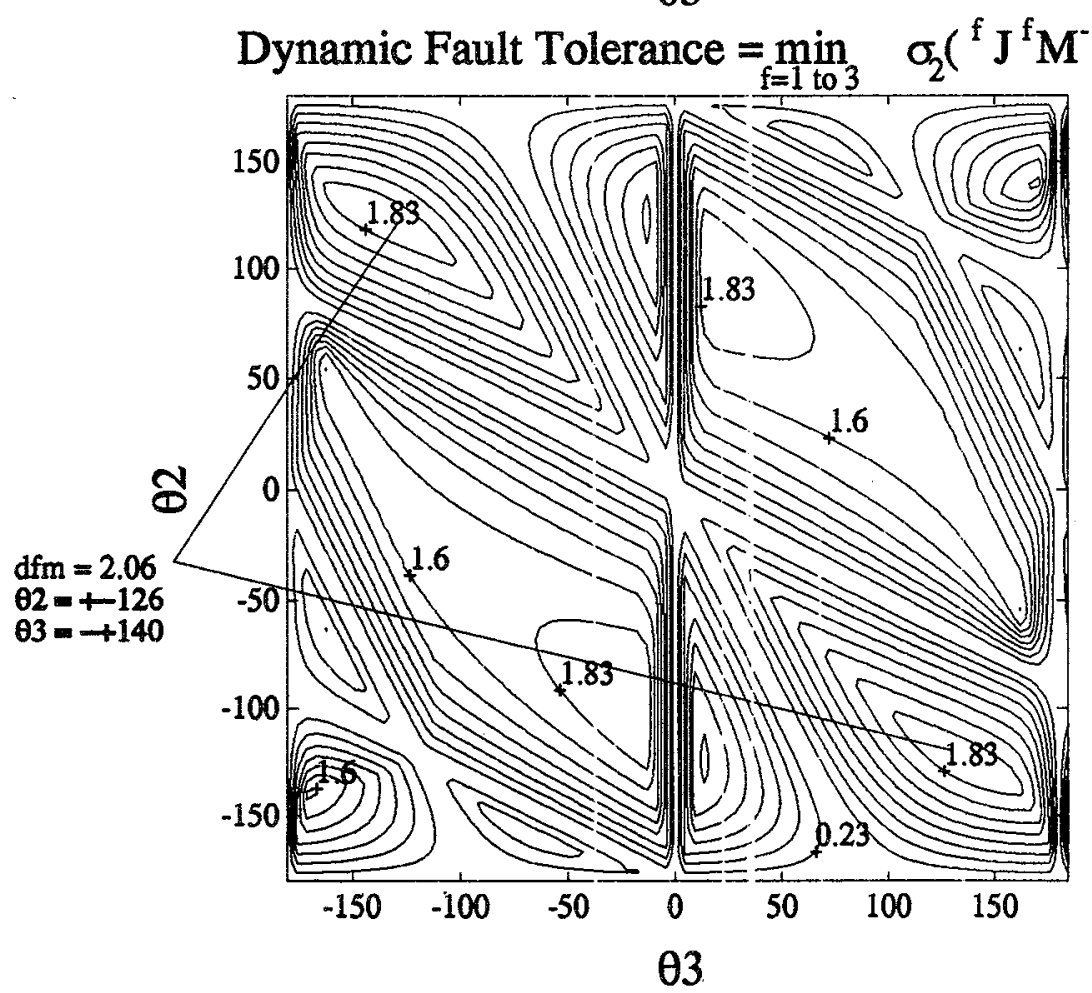

Fig. 3. The dynamic dexterity and fault tolerance measures for a 3 DOF planar manipulator are plotted as a function of the manipulator's configuration. In this case, the manipulator's links are all $1 \mathrm{~m}$ long, however, the mass of the first link is $0.8 \mathrm{~kg}$ whereas the second and third links are both of mass $0.1 \mathrm{~kg}$.

(Compare to Figs 1 and 2.)

The kinematic and dynamic dexterity measures for the first mass ratio case are shown in Fig. 1. The minimum for both measures occurs at the reach singularity which is indicated by the zero at the origin of both plots. Clearly, the zeros of the dynamic dexterity measure must match those of the kinematic measure since they are both due to a rank deficiency in $J$. The effect of $M^{-1}$ in the definition 
of $d m$ is basically limited to skewing and stretching the contours around these fixed minima. It is interesting to note, however, that the maxima for both measures are also relatively close to each other. The manipulator configurations for these maxima are illustrated in Fig. 4(a) and (c).

The kinematic and dynamic fault tolerance measures for this manipulator are shown in Fig. 2. By comparing Fig. 1 with Fig. 2, the most striking feature is that the point minimum at the reach singularity has been expanded to include several lines of minima that partition the joint space. Physically, these minima represent all of the singularities of the resulting two-link manipulators that can occur after an arbitrary joint failure so that it is easy to geometrically describe the conditions that these minima represent. For example, the singularity which occurs on the line $\theta_{3}=0$ is due to a failure of the first joint, which puts the resulting two-link manipulator into a reach singularity since $j_{2}$ and $j_{3}$ are co-linear. Likewise if $\theta_{3}=-2 \theta_{2}$ then the end effector lies on the same line as the first link and $j_{1}$ and $j_{2}$ are co-linear, thus resulting in a singularity if the third joint fails. A similar geomtric argument describes the lines of singularities associated with the loss of the second joint.

The existence of so many minima that partition the joint space has a profound effect on the resulting dynamic fault tolerance. Since the zeros of these two measures must match, there is a far less pronounced skewing effect due to $M^{-1}$ when calculating $\mathrm{dfm}$. Thus for all practical purposes the manipulator is restricted to a small region of the joint space if it is to maintain some level of fault tolerance. It is interesting to note, however, that the globally optimal dynamic fault tolerant configuration is not within the same partition of joint space as the globally optimal kinematic fault tolerant measures [see Fig. 4(b) and (d)]. However, this is a minor point since the local maxima in each region are comparable. It is also interesting to note that despite the radical difference between the countours of $\mathrm{km}$ and $\mathrm{kfm}$, both of their optimal configurations occur at the same point, which is isotropic [6,7] [see Fig. 4(a) and (b)]. This is fortunate since one would not like to sacrifice the current normal performance of the manipulator in anticipation of a future joint failure.

Finally, Fig. 3 illustrates the effect of changing the mass distribution on the dynamic dexterity and fault tolerance measures. Since the link lengths are the same as in the previous case, the kinematic measures are identical to those in Figs 1 and 2. The contours of $d m$ are now quite different from the previous case, however, the optimal configuration has not substantially changed [see Fig. 4(c) and (e)]. Moving more of the mass towards the base of the manipulator tends to reduce the effect of the dynamics and therefore makes $\mathrm{dm}$ take on more of the characteristics of $\mathrm{km}$. The same is true for the dynamic fault tolerance measure although the lines of singularities are still by far the most defining feature.

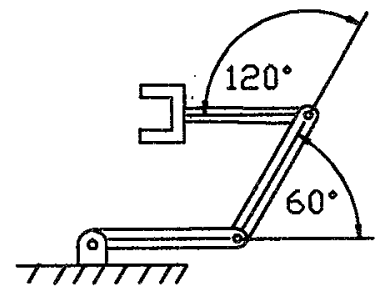

a. Dptimal $\mathrm{km}$

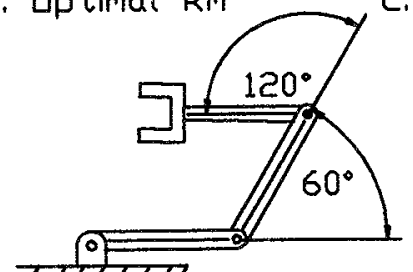

7भा77

b. Dptimal $\mathrm{kfm}$

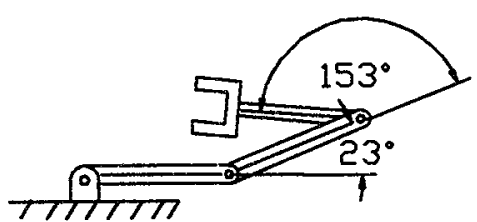

c. Dptimal dm, Case 1

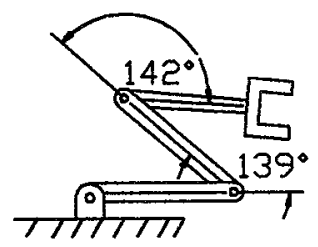

d. Dptimal dfm, Case1

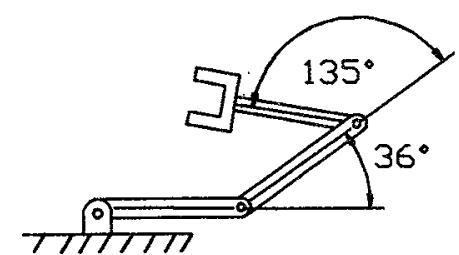

e. Iptimal $\mathrm{dm}$, Case 2

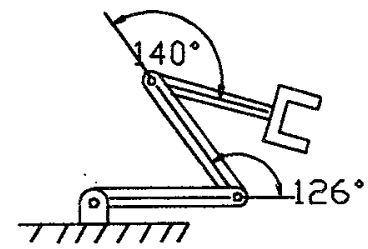

f. Dptimal dfm, Case 2

Fig. 4. Configurations (a) and (b) have the maximum kinematic dexterity and fault tolerance, respectively, for the manipulator described in Fig. 1. Configurations (c) and (d) have the maximum dynamic dexterity and fault tolerance, respectively, for this same manipulator. Configurations (e) and (f) have the maximum dynamic dexterity and fault tolerance, respectively, for the manipulator described in Fig. 3. Although, the configurations of optimal dynamic fault tolerance are markedly different from the optimal kinematic fault tolerant configuration, locally optimal dynamic fault tolerant configurations exist near the optimal 


\section{CALCULATION OF AN INVERSE}

In the previous section, globally optimal fault tolerant configurations were identified. These configurations are points in the $\theta_{2}-\theta_{3}$ plane, and lines in c-space, but are not, in general sufficient for tracking arbitrary trajectories in the workspace. In this section, an inverse kinematic function is developed for tracking arbitrary trajectories in the workspace. This inverse kinematic function will prove to have desirable properties prior to a failure, as well as providing maximum usability after a failure occurs.

To derive the optimal inverse kinematic function for the 3 DOF manipulator discussed in the previous section, it is advantageous to decompose the Cartesian position, $x \in R^{2}$, of the end effector into its polar form where:

and

$$
r=\sqrt{x_{1}^{2}+x_{2}^{2}}
$$

$$
\phi=\tan ^{-1}\left(\frac{x_{2}}{x_{1}}\right) .
$$

Then, because $r$ in this case is not a function of $\theta_{1}$, the inverse kinematic function, $f^{-1}$ is decomposed as follows:

$$
\begin{aligned}
{\left[\begin{array}{l}
\theta_{2} \\
\theta_{3}
\end{array}\right] } & =f_{2,3}^{-1}(r) \\
\theta_{1} & =f_{1}^{-1}\left(\phi, \theta_{2}, \theta_{3}\right)
\end{aligned}
$$

where $f_{2,3}^{-1}(r)$ is designed to select the optimal configuration from the set of $\theta_{2}$ and $\theta_{3}$ values which put the end effector at the desired radius and $f_{1}^{-1}\left(\phi, \theta_{2}, \theta_{3}\right)$ in effect chooses $\theta_{1}$ to achieve the desired $\phi$ given the values of $\theta_{2}$ and $\theta_{3}$. Families of curves in the $\theta_{2}-\theta_{3}$ plane which yield a given radius are shown as dotted contour lines in Fig. 5. Along each constant radius contour in $\theta_{2}-\theta_{3}$ space,

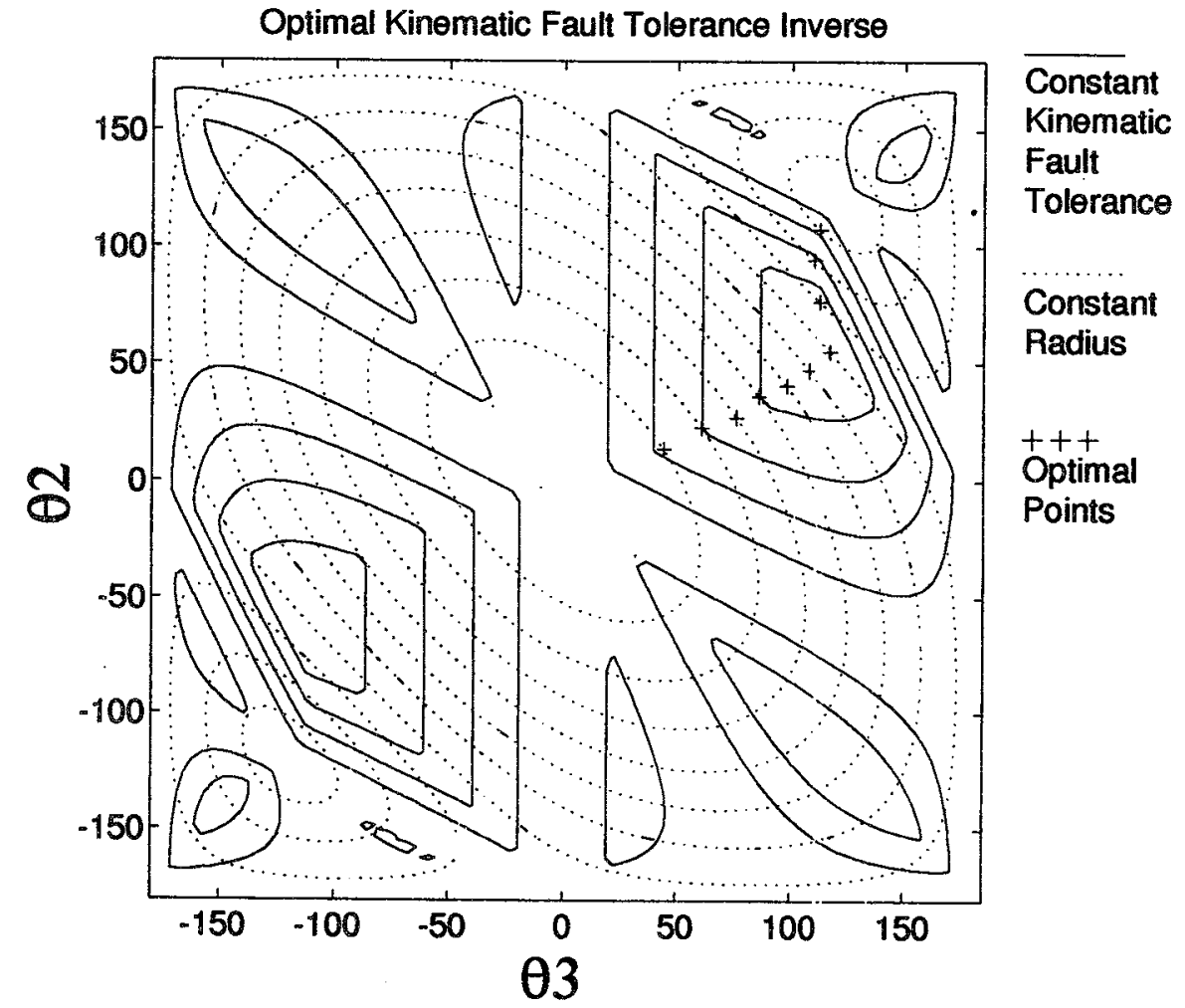

Fig. 5. The self-motion curves for the 3 DOF manipulator described in Fig. 1 are depicted with dotted lines superimposed on the contours of constant fault tolerance. The points optimizing the kinematic fault tolerance along each of these curves can be used to define an optimally fault tolerant inverse kinematic function. 
the value of $\mathrm{kfm}$ varies and thus may be optimized. A sampling of the optimal $\mathrm{kfm}$ points along each radius is given in Fig. 5 . The optimal points all lie in the same singularity-bounded region which lies predominantly in the first quadrant. The symmetric third quadrant singularity-bounded region has identical optimal points which are not noted. Optimal points typically lie on ridges of the $k f m$ function which occur where two of the ${ }^{f_{J}}$ have identical minimum singular values. The desired inverse, $f_{2,3}^{-1}(r)$, is the set of all points maximizing $\mathrm{kfm}$ parameterized by $r$, and is depicted as a bold curve in Fig. 6 . The curve, $f_{2,3}^{-1}(r)$, begins at the origin where the end effector is completely stretched out to a radius of $3 \mathrm{~m}$. As the radius decreases, the inverse follows the ridge defined by the equation $\sigma_{2}\left({ }^{1} J\right)=\sigma_{2}\left({ }^{3} J\right)$ up to the optimal $\mathrm{kfm}$ point where $\sigma_{2}\left({ }^{1} J\right)=\sigma_{2}\left({ }^{2} J\right)=\sigma_{2}\left({ }^{3} J\right)$ and $r=1$. Here, the curve changes direction and follows the $\sigma_{2}\left({ }^{2} J\right)=\sigma_{2}\left({ }^{3} J\right)$ ridge down to the $\theta_{2}=\theta_{3}=120$, $r=0$ point. In Fig. 6 the contours of constant $\mathrm{kfm}$, and the inverse kinematic function $f_{2,3}^{-1}(r)$ are mapped into the workspace with $\theta_{1}$ fixed at 0 . Each singularity-bounded region is distinguished by a distinctive set of line types. In the figure, varying $\theta_{1}$ would simply rotate the mapping about the origin, therefore, it is the range of $r$ spanned by a particular contour that determines the region reachable by the manipulator while operating on the given $k f m$ contour. Once the inverse, $f_{2,3}^{-1}$, has been computed, it is straightforward to compute $\theta_{1}$ using:

$$
\begin{aligned}
\theta_{1} & =f_{1}^{-1}\left(\phi, \theta_{2}, \theta_{3}\right) \\
& =\phi-\tan ^{-1}\left(\frac{\sin \left(\theta_{2}\right)+\sin \left(\theta_{2}+\theta_{3}\right)}{1+\cos \left(\theta_{2}\right)+\cos \left(\theta_{2}+\theta_{3}\right)}\right) .
\end{aligned}
$$

It was very fortuitous that, in the kinematic case, the set of optimal points parameterized by $r$ formed a continuous curve within a single region. It is possible that the optimal points for different values of $r$ occur in different singularity-bounded regions. When this occurs, one can construct a continous inverse from points that are locally optimal and lie within a single region. The manipulator dynamics, as shown in Fig. 2, represents a non-linear weighting on the kinematic fault tolerant function. This stretching of the $\mathrm{kfm}$ function causes the $f_{2,3}^{-1}(r)$ curve of optimal $\mathrm{dfm}$ points parameterized by $r$ to jump from one region to another as $r$ varies. In Fig. 7 each of the regions labeled $A, B$ and $C$ contain portions of the set of optimal $d f m$ points. Figure 7 displays two different dynamic fault tolerant inverses that were constructed from locally optimal $\mathrm{dfm}$ points, one through the region labeled $A$, and the other through the regions labeled $B$ and $C$. Either of the curves is a plausible inverse kinematic function.

In Fig. 7, the associated workspace maps for the $d f m$ contours and both plausible $f_{2,3}^{-1}(r)$ curves are drawn. Since both of the functions have segments of global optimality and segments of only local optimality, the question naturally arises as to which is a more appropriate inverse function. There are several factors affecting the choice of $f_{2,3}^{-1}(r)$ that one should consider. One obvious benefit of the inverse function from region $A$ is that it spans the entire workspace within a single region, while the inverse function from regions $B$ and $C$ passes through a line of $d f m=0$ when it crosses from the $B$ to the $C$ region. However, in certain applications, all of the trajectories may lie outside of a radius of $1 \mathrm{~m}$ and therefore, the $B-C$ boundary need not be crossed. If trajectories do cross the boundary, the time spent with low fault tolerance may be short in practice since the value of $d f m$ rises very quickly on either side of the boundary. One obvious benefit of the inverse function from the $B$ and $C$ regions is that it contains the largest value of $d f m$, therefore, if crossings of the $B-C$ boundary are unusual, an integral of $d f m$ along typical paths may favor using this inverse function. One should note, however, that on the boundary between the $B$ and $C$ regions, this inverse results in high accelerations of $\theta_{3}$. Another factor that may favor the inverse from the $B$ and $C$ regions is the even distribution of joint motion throughout the workspace. Using the inverse function from the $B$ and $C$ regions results in approximately equal amounts of motion in $\theta_{2}$ and $\theta_{3}$ for the entire workspace. The inverse from the $A$ region, on the other hand, has two distinct segments, where, in each segment, motion of one of the joints is favored over the other. For smaller values of $r$, the inverse from region $A$ results in mostly $\theta_{3}$ motion, then, for larger values of $r$ it results in mostly $\theta_{2}$ motion. This effect can be explained in physical terms by noting that the motion of the outboard links has less dynamic resistance than motion of the inboard ones and thus is preferred until the kinematic limitations require motion of the inboard links. 

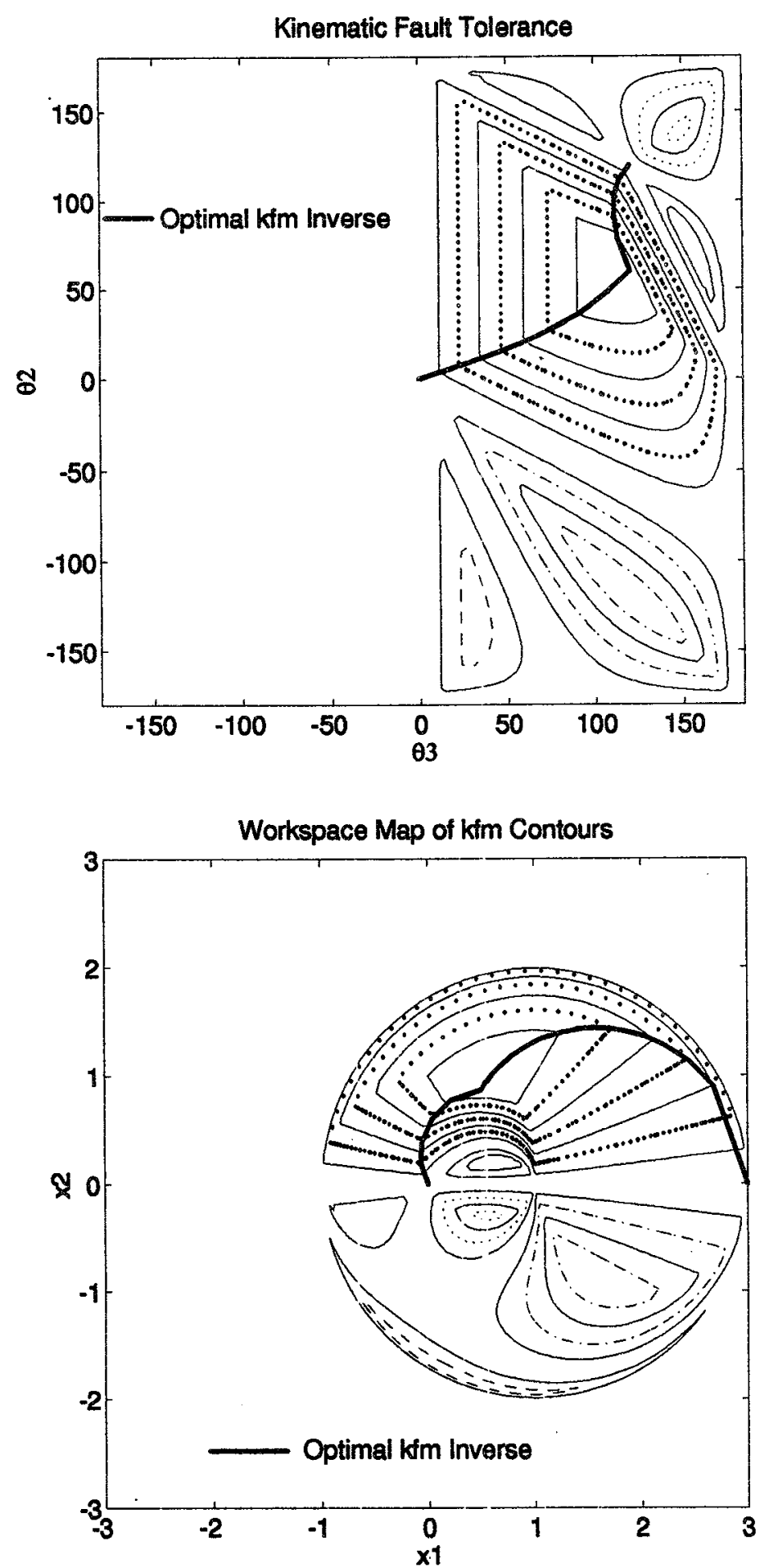

Fig. 6. The bold line depicts an optimal kinematically fault tolerant inverse function for the $3 \mathrm{DOF}$ manipulator described in Fig. 1. This function is used for determining $\theta_{2}$ and $\theta_{3}$ given the desired radial distance of the end effector from the manipulator's base. Mapping the constant kinematic fault tolerance contours and the inverse kinematic function into the workspace with $\theta_{1}=0$ indicates the regions in the actual workspace that may be swept out while maintaining a given degree of fault tolerance. 

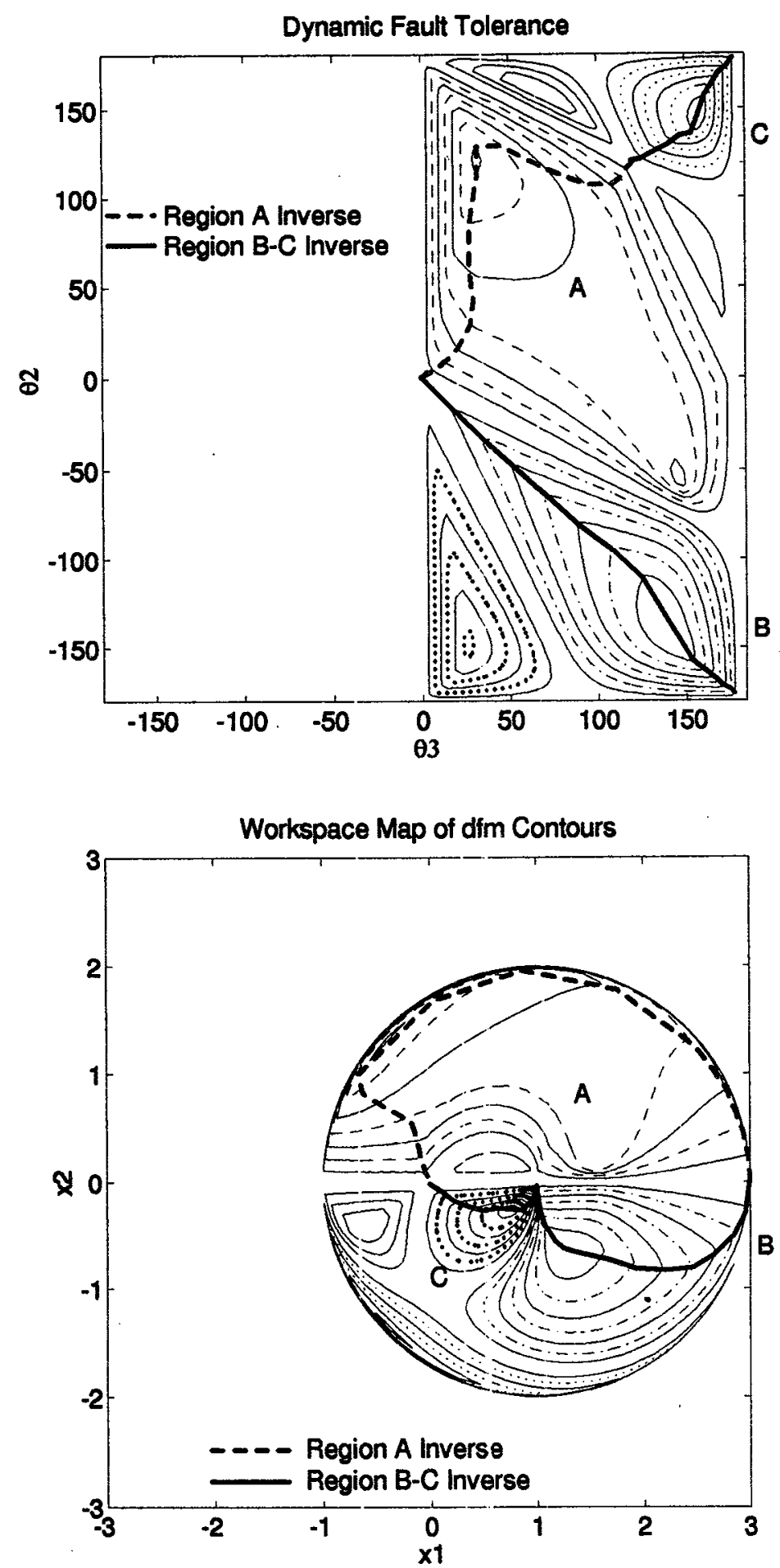

Fig. 7. The solid and dashed bold curves depict dynamically fault tolerant inverse functions for the 3 DOF manipulator described in Fig. 1. Two functions are shown since each is composed of some sections of globally maximal values of dynamic fault tolerance and other sections which are only locally optimal. This is in contrast to Fig. 6 where a single inverse function contains all of the globally optimal values.

\section{SIMULATION}

This section presents a simulation of the optimal kinematic fault tolerant inverse, described in the previous section, and evaluates its performance both before and after a failure. Its performance is compared to pseudoinverse control along the test trajectory depicted in Fig. 8. In the simulation, 


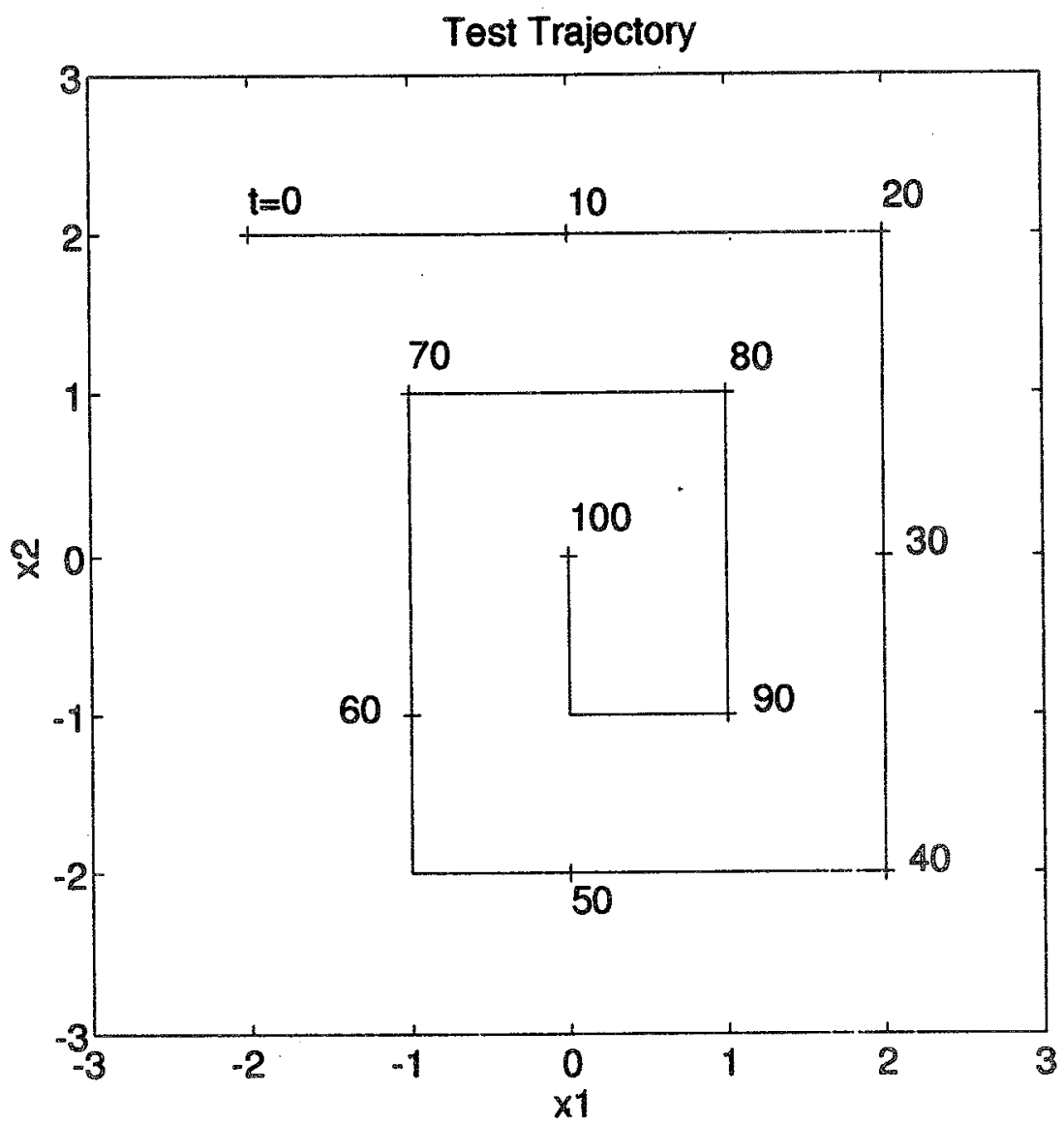

Fig. 8. The desired end-effector trajectory used to evaluate the performance of the optimal kinematic fault tolerant inverse. The norm of the desired end-effector velocity is constant thus resulting in directional discontinuities at the corners.

the manipulator follows the trajectory with a constant linear velocity as it spirals toward the origin. The path was selected to cover virtually the entire workspace, and to have a wide range of ratios of $\dot{\phi}$ and $\dot{r}$. It is discontinuous in direction at the corners to help distinguish points along the trajectory and to emphasize the directional nature of the inverses. The trajectory also passes through the point $x=[0,1]^{T}$ which will result in the globally optimal fault tolerant configuration when the kinematic fault tolerant inverse function is used.

Figure 9 compares the norms of the joint velocities resulting from the two inverses along the trajectory. Note the similarity of the norm of the joint velocity resulting from the optimal $\mathrm{kfm}$ inverse to that of the pseudoinverse for the first half of the trajectory. This is expected since the optimal $\mathrm{kfm}$ inverse moves nearly perpendicular to the curves of constant radius for most of its length (see Fig. 9). Constant radius curves are projections of the self-motion curves in $c$-space onto the $\theta_{2}-\theta_{3}$ plane. The pseudoinverse, by definition, results in trajectories that are orthogonal to the self-motion curves. In the second half of the trajectory, the optimal $\mathrm{kfm}$ inverse has even lower joint velocities than that of the pseudoinverse. This effect is also expected, since the configurations have diverged and because the configuration resulting from the pseudoinverse is more ill-conditioned than the one resulting from the optimal $\mathrm{kfm}$ inverse.

In general, high joint velocities are to be expected when operating near the boundary of the workspace if the desired end-effector velocity contains a component in the $\dot{r}$ direction. This effect is responsible for the $U$ shaped segments of Fig. 9 between the times $t=0-20,20-40$ and 40-55 of the trajectory. In these regions, the direction of the desired end-effector velocity varies from having equal components of $r$ and $\phi$ to being strictly along $\phi$. A related effect occurs when the manipulator is in a poorly conditioned configuration. In such configurations, sharp changes in the 


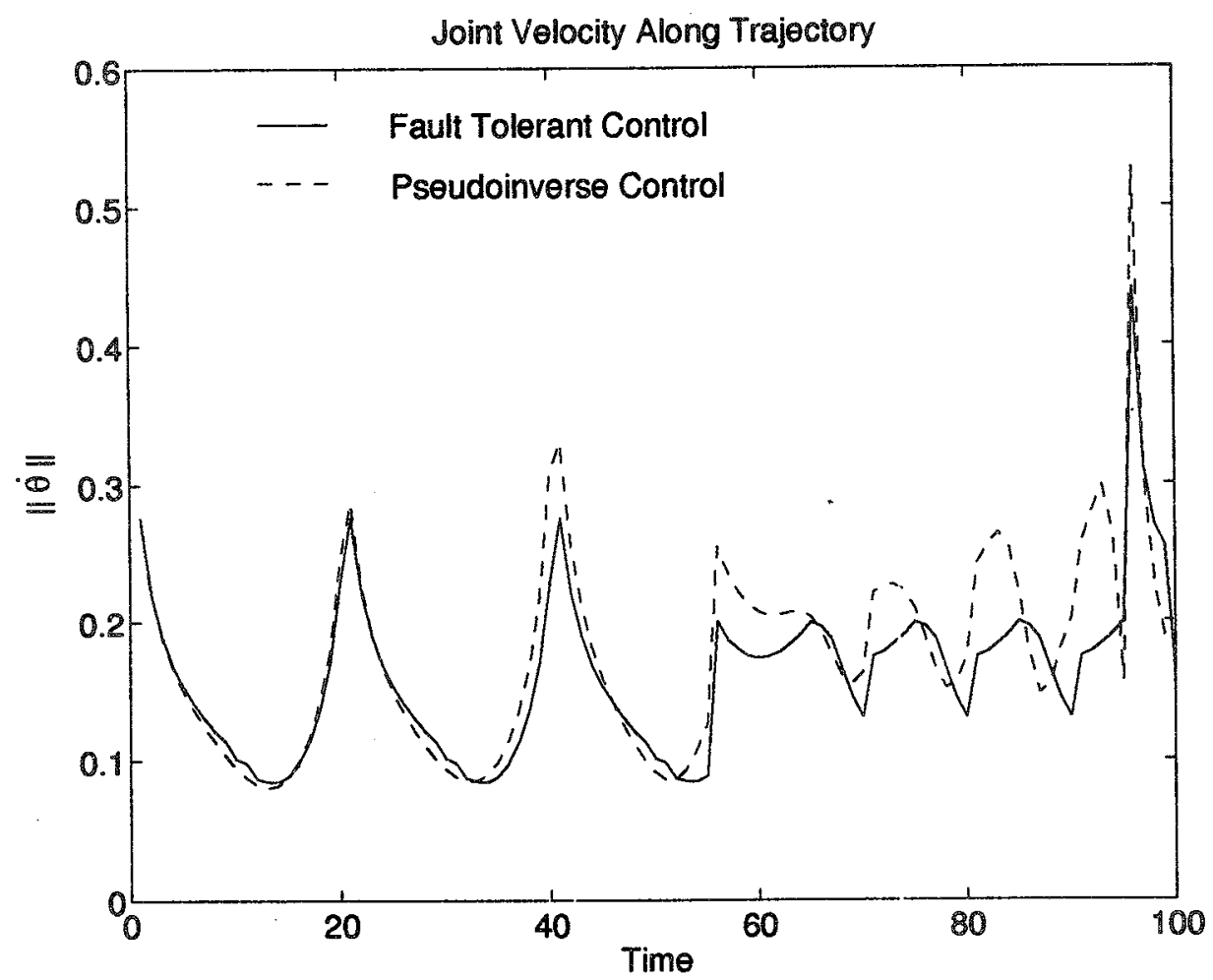

Fig. 9. The norm of the joint velocity for following the trajectory described in Fig. 8 is shown for both the pseudoinverse and the optimal kinematic fault tolerant inverse. Note that the discontinuities are due to those present in the desired end-effector trajectory and can be used to infer the directional dependence of the inverses.

direction of motion are marked by sharp differences in the norm of the joint velocity. For example, immediately before $t=95$, most of the motion was in the $\phi$ direction whereas at $t=95$, the motion switches to being predominantly in the $r$ direction. Both inverses mark this switch with a pronounced discontinuity in $\|\dot{\boldsymbol{\theta}}\|$. On the other hand, even though the direction of the trajectory changes at times $t=20$ and $t=40$, no significant discontinuity in the norm of the joint velocity occurs since approximately equal ratios of $\dot{r}$ and $\phi$ are necessary both before and after the turn.

The above discussion shows that the optimal $\mathrm{kfm}$ inverse results in well-conditioned configurations, however, the real benefits of using the optimal $\mathrm{kfm}$ inverse over the pseudoinverse become apparent when one considers operation after a joint failure. To evaluate the performance of each inverse after a failure, all possible single joint failures are simulated to occur at $t=75\left(x=[0,1]^{T}\right)$ on the test trajectory. The resulting failure configurations for both of the inverses are shown in Fig. 10 along with the remaining path which is indicated with a bold line. First, consider locking the first joint while the second two joints remain usable. The remaining region of the workspace that is reachable by the resulting manipulator for both cases is a circle of radius $2 \mathrm{~m}$ centered at the end of the first link and is indicated with a dotted line. Note that in the pseudoinverse case, a portion of the remaining trajectory lies outside of the new workspace boundary while in the resulting optimal $\mathrm{kfm}$ configuration the entire remaining path is reachable by the failed manipulator. Next, consider the configurations with the second joint locked and the first and third joints functioning. The resulting boundaries of the reachable workspace with the second joint failing are drawn with dashed lines. For the pseudoinverse case the remaining path is inside the resulting reachable region except for a very small segment at the end. The same is true for the resulting optimal $\mathrm{kfm}$ configuration except that the unreachable segment of the path is slightly longer. Finally, consider the third joint failing. This time, the inner and outer boundaries are drawn with solid circles centered at the origin. The remaining workspace resulting from using the optimal $\mathrm{kfm}$ inverse has no inner boundary and an outer boundary of a $2 \mathrm{~m}$ radius and thus can complete the 


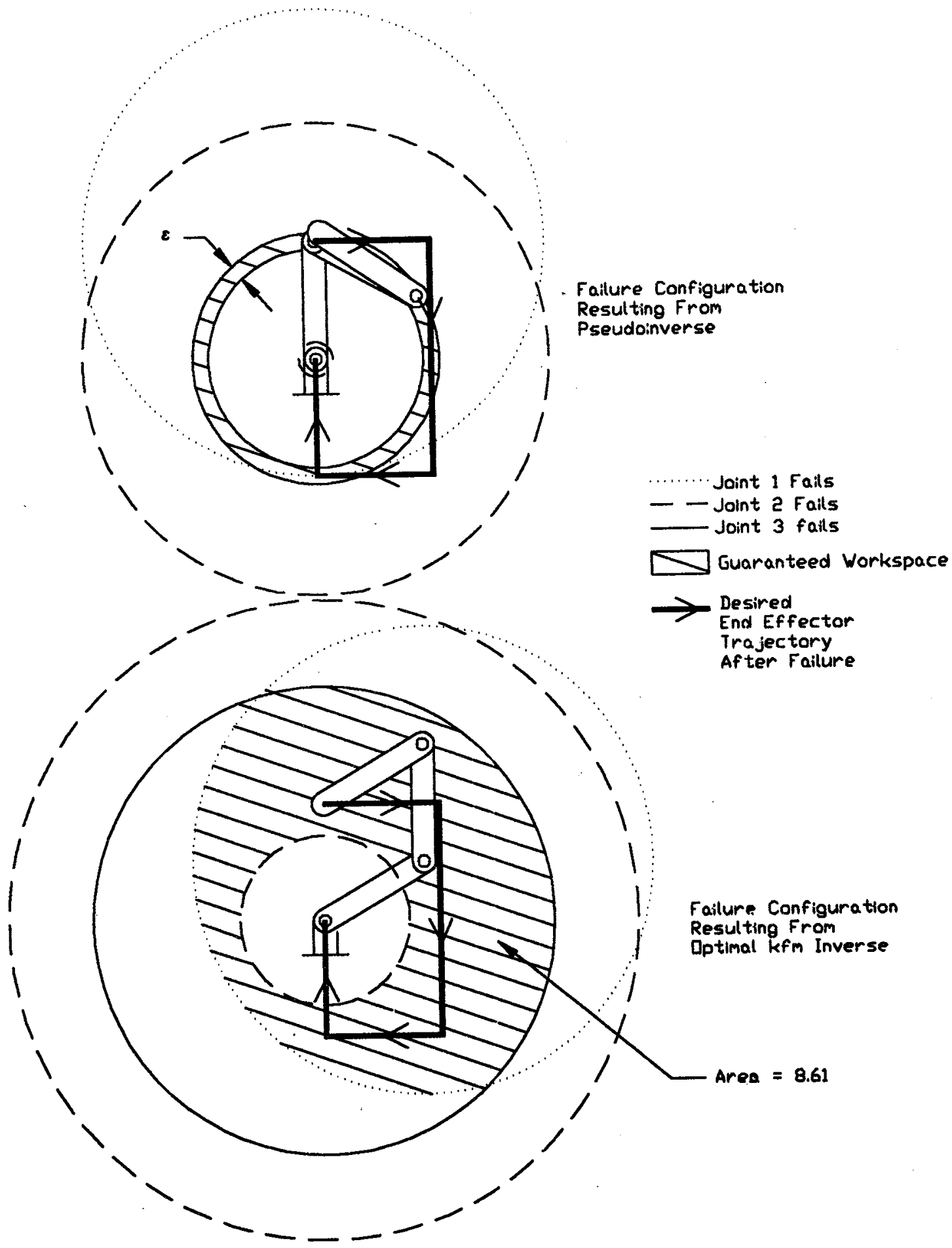

Fig. 10. These configurations resulted from using the pseudoinverse and the optimal kinematic fault tolerant inverse on the test trajectory described in Fig. 8 up to the positions shown. Circles delineate the remaining reachable workspace should a particular joint fail while the manipulator is at this configuration. The shaded regions are reachable by the manipulator from these configurations irrespective of which joint fails. The configuration resulting from the pseudoinverse clearly does not possess a high degree of fault tolerance since a failure of the third joint would cause the manipulator to immediately be unable to proceed along the path. 
entire trajectory. The failure configuration resulting from the pseudoinverse has the third link folded in on the second link and thus the failed manipulator has an effective reachable region that consists of only a circle of $\epsilon$ thickness at a radius of $1 \mathrm{~m}$. The width $\epsilon$ will, in fact, approach zero as $\theta_{3}$ approaches $\pi$. It should be clear that if the third joint fails in this configuration the manipulator will immediately lose it's ability to track the desired path. In summary, if either the first or third joint fails, only the configuration resulting from using the optimal $\mathrm{kfm}$ inverse would be able to complete the task while if the second joint fails neither of the resulting configurations allow the task to be completed.

For any given configuration, the region of the workspace that remains reachable under all three of the possible single joint failures in the manipulator is the guaranteed reachable workspace. In Fig. 10 the guaranteed reachable workspaces are shaded for both of the configurations that result at $t=75$ from using the two different inverses. Since the optimal $\mathrm{kfm}$ inverse function maximizes the minimum singular value of the Jacobians resulting from failures, and because the minimum singular value is a measure of the distance from a singularity, the optimal $\mathrm{kfm}$ inverse function keeps the manipulator away from what would be singularities if a failure were to occur. Since workspace boundaries are by definition singular configurations, using the optimal $\mathrm{kfm}$ inverse insures that the manipulator will be in a configuration that is away from a workspace boundary should a joint fail. In a global sense, one goal of fault tolerance is to have the guaranteed workspace at each point along the desired trajectory contain the rest of the trajectory. This criterion is very path specific and attempting to maintain it may require unnecessarily restricting the usable region of the workspace for the manipulator prior to a failure. The optimal $\mathrm{kfm}$ inverse function has the desirable properties that it does not restrict the usable workspace in anticipation of a fault, it maintains a relatively high dexterity before a failure, and it guarantees at least local tracking after a failure occurs.

\section{CONCLUSIONS}

This work has extended the notions of kinematic and dynamic dexterity to define measures of kinematic and dynamic fault tolerance for kinematically redundant robotic manipulators. These measures were then used to identify operating regions in which the manipulator remains usable even when one of its joints must be locked due to a failure. It was shown that the minima of the kinematic fault tolerant measure were the single most important factors in defining such regions. An optimal fault tolerant inverse kinematic function was then calculated and shown to not only provide maximal dexterity in the event of a failure, but also to have desirable performance before the occurrence of a failure.

Acknowledgements - This work was supported by Sandia National Laboratories under contract No. 18-4379B. Additional funding was provided by the National Science Foundation under grant CDR 8803017 to the Engineering Research Center for Intelligent Manufacturing Systems.

\section{REFERENCES}

1. H. Arai and S. Tachi, Position control of a manipulator with passive joints using dynamic coupling. IEEE Trans. Robot. Automat. 7, 528-534 (1991).

2. B. Christensen, W. Drotning and S. Thunborg, Model-based, sensor-directed remediation of underground storage tanks. J. Robot. Sys. 9, 145-159 (1992).

3. E. Papadopoulos and S. Dubowsky, Failure recovery control for space robotic systems. In 1991 American Control Conference, pp. 1485-1490. Boston, Mass. (1991).

4. R. Colbaugh and M. Jamshidi, Robot manipulator control for hazardous waste-handling applications. J. Robot. Sys. 9, 215-250 (1992).

5. A. A. Maciejewski, The design and control of fault tolerant robots for use in hazardous or remote environments. In Robotics and Remote Systems: Proceedings of the Fourth American Nuclear Society Topical Meeting on Robotics and Remote Systems (Edited by M. Jamshidi and P. J. Eicker), pp. 633-642. Albuquerque, N.M. (1991).

6. C. A. Klein and T. A. Miklos, Spatial robotic isotropy. Int. J. Robot. Res. 10, 426-437 (1991).

7. J. Angeles, The design of isotropic manipulator architectures in the presence of redundancies. Int. J. Robot. Res. 11, 196-201 (1992).

8. T. Yoshikawa, Manipulability of robotic mechanisms. Int. J. Robot. Res. 4, 3-9 (1985).

9. T. Yoshikawa, Dynamic manipulability of robot manipulators. J. Robot. Sys. 2, 113-124. (1985). 


\section{AUTHORS' BIOGRAPHIES}

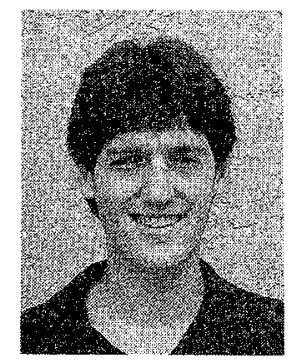

Christopher L. Lewis - Christopher L. Lewis received a B.S. degree in mechanical engineering from Kansas State University, Manhattan, in 1987 and an M.S. degree in electrical engineering from Purdue University, West Lafayette, Ind. in 1990. He is currently a Ph.D. candidate at Purdue University.

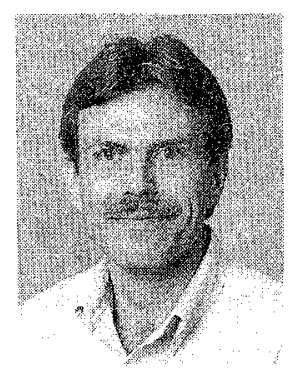

Anthony A. Maciejewski-Anthony A. Maciejewski received the B.S.E.E., M.S., and Ph.D. degrees in electrical engineering from The Ohio State University, Colombus, in 1982, 1984, and 1987 respectively. Since 1988 he has been with the School of Electrical Engineering at Purdue University, West Lafayette, Ind., where he is currently an Associate Professor. His primary research interests center on the simulation and control of kinematically redundant robotic systems. 